\section{Non-invasive imaging demonstrates clinical features of ankylosing spondylitis in a rat adjuvant model: a case study}

\author{
N. Accart, ${ }^{1}$ J. Dawson, ${ }^{2}$ F. Kolbinger, ${ }^{2}$ \\ I. Kramer, ${ }^{1}$ N. Beckmann'
}

'Musculoskeletal Diseases Department, Novartis Institutes for BioMedical Research, Basel

${ }^{2}$ Autoimmunity, Transplantation and Inflammation Department, Novartis Institutes for BioMedical Research, Basel, Switzerland

\section{Abstract}

Main features of ankylosing spondylitis like inflammatory erosive osteopenia and bony overgrowth are recapitulated in rats challenged with complete Freund's adjuvant. In vivo changes induced in the rat spine were followed longitudinally by magnetic resonance imaging (MRI) and assessed terminally by micro-computerized tomography (micro-CT) and histology. Signals reflecting inflammation were detected by MRI at levels L5-L6 throughout the experiment, peaking at day 27 after adjuvant. Bone erosion and formation occurred from this time point onward, as confirmed by micro-CT. Histology confirmed the inflammation and bone remodeling. The present study demonstrates the potential of imaging for longitudinal assessments of spinal changes in this animal model and the excellent correlation between in vivo images and histology underlines its fundamental role in the validation of non-invasive imaging.

\section{Introduction}

Ankylosing spondylitis (AS) is a common rheumatic disease with a prevalence of $0.9 \%$ worldwide. ${ }^{1}$ The mechanism of pathogenesis is poorly understood despite the recognition of the role of the human leukocyte antigen B27 (HLA-B27) in $90-95 \%$ of AS patients, and the interleukin 23 receptor (IL-23R) polymorphism. ${ }^{2,3}$ AS involves both inflammatory erosive osteopenia and unusual bony overgrowth gradually replacing joints tissue. ${ }^{4}$ When lesions occur in the spine, the ultimate bone remodeling occurs with complete vertebrae fusion. The delay between onset of symptoms and diagnosis can reach up to 10 years, ${ }^{5}$ incur- ring significant disability and economic cost. ${ }^{6}$

Currently, the most effective medication for AS encompasses biologic agents blocking TNF- $\alpha{ }^{7,8}$ The ability of these agents to prevent ankyloses remains controversial, despite the fact that recent long-term studies provided encouraging results. ${ }^{8,9}$ The development of different animal models have been useful for the study of AS pathogenesis and for testing of new therapeutic concepts. Progressive ankyloses in ank/ank mice, HLA-B27 transgenic mice and rats, as well as challenges with complete or incomplete Freund's adjuvant associated with proteoglycans have been explored. ${ }^{10-14}$

Imaging techniques aim at bridging preclinical and clinical studies. It has been shown that computerized tomography (CT) and magnetic resonance imaging (MRI) are better techniques for the detection of earlier AS features in humans than radiography. ${ }^{15-17} \mathrm{We}$ applied MRI to follow longitudinally in vivo, at the level of the rat spine, changes induced by complete Freund's adjuvant (CFA) inoculation into the tail. ${ }^{18,19}$ Post-mortem analyses included micro-CT imaging and histology of the isolated spine. This study shows interesting findings in imaging readouts and reiterate the fundamental role played by histology in order to validate non-invasive techniques.

\section{Materials and Methods}

Experiments were performed according to animal license number BS-1505, granted by the Veterinary Authority of the City of Basel, Switzerland.

\section{Animals and disease induction}

Han Wistar rats ( $\mathrm{n}=2$, Charles River Laboratories, Wilmington, MA, USA) were acclimatized in a controlled environment for one week. Polyarthritis was induced in one rat by an intracutaneous injection at the tail base, of 0.1 $\mathrm{mL}$ of CFA [6 mg Mycobacterium tuberculosis (H37RA, DIFC0, Becton Dickinson) suspended in paraffin oil $(1 \mathrm{~mL})] \cdot{ }^{20}$ The other animal served as control. Animals were imaged from the second week following CFA injection, when the paws were already inflamed. The study was finished on day 60 after CFA.

\section{Magnetic resonance imaging}

For MRI, rats were anesthetized with 2.0\% isoflurane (Abbott, Cham, Switzerland) in a mixture of $\mathrm{O}_{2} / \mathrm{N}_{2} \mathrm{O}(2: 1)$, administered via a nose cone. Body temperature was kept at $37 \pm 1^{\circ} \mathrm{C}$ using a heating pad. Measurements were carried out with a Biospec 70/30 spectrometer (Bruker Medical Systems, Ettlingen, Germany) operating at $7.0 \mathrm{~T}$. A quadrature resonator with $70 \mathrm{~mm}$ inner diameter (Bruker) was used for signal
Correspondence: Nathalie Accart,

Musculoskeletal Diseases/Imaging and Histology, Novartis Institutes for BioMedical Research, Forum 1, Novartis Campus, Fabrikstrasse 28-3.04.01, CH-4056 Basel, Switzerland.

Tel. +41.796.734877.

E-mail: nathalie.accart_gris@novartis.com

Key words: Ankylosing spondylitis; histology; histochemistry; magnetic resonance imaging (MRI); micro computerized tomography (micro-CT).

Contributions: NA, all histological analyses; JD, development of the model and managing of animal experiment; FK, leading of the ankylosing project; IK, micro-CT; NB, MRI acquisitions and study coordination.

Conflict of interest: the authors declare that no conflicts of interest were handled during the planning, implementation, writing, peer review, editing, and publication of this scientific work.

Acknowledgments: we are indebted to Nella Vidotto, Christelle Gerard, Bernhard Jost, Marcel Merdes, Charles Moes, Peter Ingold and Stefan Zurbruegg for technical support.

Received for publication: 21 April 2016.

Accepted for publication: 22 September 2016.

This work is licensed under a Creative Commons Attribution-NonCommercial 4.0 International License (CC BY-NC 4.0).

CCopyright N. Accart et al., 2016

Licensee PAGEPress, Italy

European Journal of Histochemistry 2016; 60:2667 doi:10.4081/ejh.2016.2667

excitation and detection. Three-dimensional (3D) gradient-echo imaging (see Figure 2 legend for parameters) was started 6 min after i.v. injection of Gd-DOTA (Guerbet, Aulnay-sous-Bois, France; $0.15 \mathrm{~mL}$ ) as a bolus into the tail vein. ${ }^{21}$

\section{Post-mortem analyses}

At the end of the study animals were euthanized by an overdose of thiopental (Pentothal ${ }^{\circledR}$, Abbott; $250 \mathrm{mg} / \mathrm{kg}$ i.p., $0.2 \mathrm{~mL}$ ) and the spines were excised for micro-CT and histological examination. Spine samples were first fixed in $4 \%$ neutral buffered paraformaldehyde for two days and then kept in $70 \%$ ethanol at $4^{\circ} \mathrm{C}$ until completion of micro-CT.

\section{Micro computerized tomography}

Scans of fixed spine were performed on a uCT40 instrument (Scanco Medical, Brüttisellen, Switzerland) using the following parameters: energy/intensity: $70 \mathrm{kVp}, 57 \mu \mathrm{A}, 4 \mathrm{~W}$; image matrix: 2048x2048 pixels, voxel size: 20 $\mu \mathrm{m}$. Segmentation thresholds of 190 and 280 and a Gaussian filter ( $\sigma=0.8$, support of one voxel) were used for image analysis. 


\section{Histology}

Following micro-CT imaging, samples were kept in a decalcification solution (ImmunoCal $^{\mathrm{TM}}$, Decal Chemical Corporation, Suffern, NY) for 14 days until decalcification was complete as verified chemically using a procedure previously described..$^{22}$ After sample dehydration and paraffin embedding, 5 - $\mu \mathrm{m}$ thick sections were cut and stained with Hematoxylin and Eosin (HE). Subsequently, additional stainings were performed to distinguish cartilage, bone, muscle, fibrotic tissue and monocytes/osteoclasts. Cartilage was stained by the Safranin 0/Fast green procedure adapted from Kalscheur. ${ }^{23}$ Safranin-0 stained the cartilaginous areas until the tidemark, while Fast green stained the subchondral mineralized bone. The Aldehyde-FuchsinPicrosirius red (AFPS) staining was used to differentiate cartilage, collagens or bone. Under this specific staining, newly formed bone has the aspect of woven bone and polarizes in green rather than orange/red. ${ }^{24}$ Osteoclasts were detected with an anti-CD68 antibody applied on paraffin sections. ${ }^{25,26}$ After an antigen retrieval step in a $10 \mathrm{mM}$ citrate buffer (pH 6) at $98^{\circ} \mathrm{C}$, the primary monoclonal antibody anti-rat ED1 (MCA341R, Bio-Rad, Puchheim, Germany) was incubated at $0.05 \mu \mathrm{g} / \mathrm{mL}$ followed by a biotinylated secondary antibody (BA-2001, Vector Lab., Burlingame, CA) and amplified by the Avidin Biotin - HRP complex (Elite PK-6100, Vector Lab.) according to manufacturer's recommendations. After the reaction was revealed with DAB, sections were counterstained with Mayer's Hematoxylin, washed, dehydrated and mounted with Pertex.

\section{Results}

The main features of AS were observed by histology. Globally, the L5-L6 lumbar spine of the CFA rat (Figure 1 A-b) was severely affected as demonstrated by the damage of the vertebral bone. Subchondral tissues were infiltrated by immune cells like osteoclasts responsible for bone resorption (Figure $1 \mathrm{~A}-\mathrm{c}$ ), polymorphonuclear cells (Figure 1 A-d), and lymphocytes (Figure $1 \mathrm{~A}-\mathrm{e}$ ). Figure 1 B-a shows the invasion of joint space by a synovial pannus (sp) resulting from fibroblast-like synoviocyte proliferation, leading to cartilage destruction (ca) and bone invasion (bo). The remaining nucleus pulposus (np) of intervertebral disc surrounded newly-formed bone (bo) replacing the annulus fibrosus (Figure $1 \mathrm{~B}-\mathrm{b}$ ). No osteoclasts were observed in this newlyformed bone (data not shown). Figure 1 B-c displays the bony ankylosis process resulting in a newly formed bone, so-called woven bone (bo), well-observed under polarized light. This confirmed the presence of inflammation and erosion, as well as new bone formation at the levels L5-L6 of the spine of the CFA-rat.

MRI images of the longitudinal development of injury induced by CFA inoculation at the lumbar levels L5-L6 of the spine are summarized in Figure 2. High intensity signals (red arrows) reflecting inflammation have been detected throughout the study. At day 14, the inflammatory response occurred along ligaments. It expanded to nearby soft tissues and

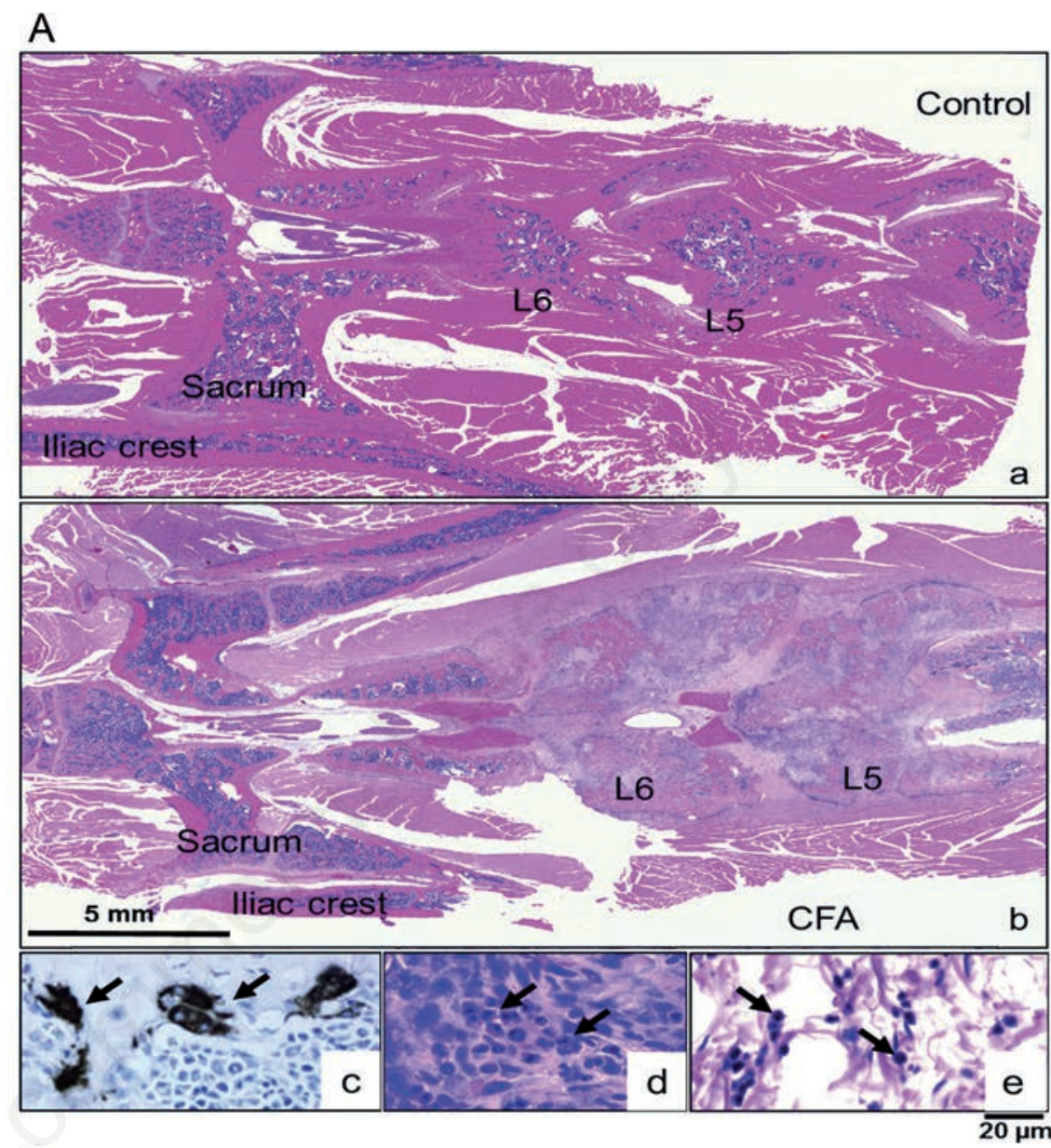

B

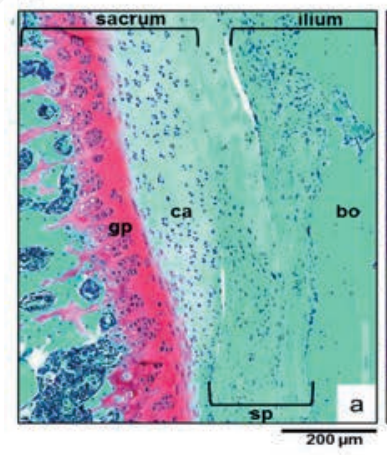

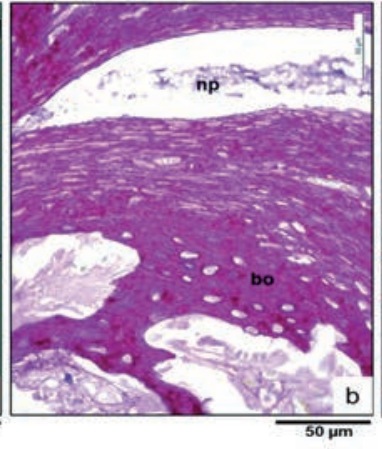

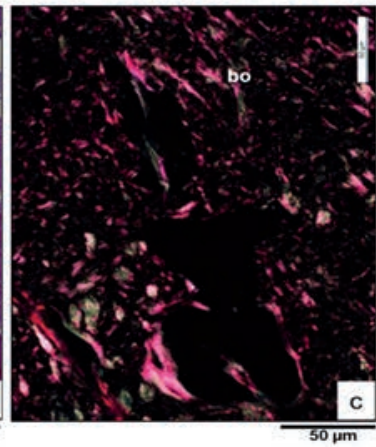

Figure 1. Histology of lumbar spine (L5-L6 level) and sacro-iliac junction. A) HE staining of the lumbar spine shows damage of the vertebral bone in a CFA rat (b) compared to the normal vertebral structure of a control animal (a); the lower panels show bone resorption by osteoclasts (dark cells in c), infiltration of polymorphonuclear cells (d), and presence of lymphocytes (e). B) Main features of the pathology developed in this CFA model; a) Safranin O/Fast green staining reveals the invasion of joint space by a synovial pannus (sp) resulting from proliferation of fibroblast-like synoviocytes; ca, cartilage; bo, bone; b) AFPS staining shows the remaining nucleus pulposus (np) of the intervertebral disc and the outer annular fibers replaced by bone (bo); c) AFPS staining coupled with the polarized light shows the new bone formation, so-called woven bone (bo). 
also to regions within the eroded bone (magenta arrows) at later time points. Moreover, images indicate the formation of bone-like structures (green arrows) from day 27 onwards.

Figure 3A presents MRI images of the lumbar spine at day 53 post-CFA, showing inflamed areas (characterized by high intensity signals) around the levels L5-L6 of the spine (red arrows) and within the bone (magenta arrows), as well as the formation of new bony structures (green arrows). Images from the control rat displayed none of these features. Post-mortem micro-CT showed severe axial bone remodeling (erosion and new bone formation) and beginning dorsal spinal fusion in the lower lumbar spine region of the CFA rat (Figure 3B). An excellent agreement was found between the new bone formation as revealed histologically and the formation of bone-like structures as indicated by in vivo MRI (Figure 3C).

\section{Discussion}

The histological data presented here indicate the potential of the rat adjuvant model to mimic some of the important human pathological features of AS, such as inflammatory response showing polymorphonuclear cells and lymphocytes as well as subchondral tissue infiltration by osteoclasts leading to bone resorption, and the disappearance of intervertebral discs and their replacement by a wellvascularized fibrotic tissue followed by ossification of fibrocartilage in the spine. This bone formation is one of the early signs of the bony fusion process of vertebrae. Using MRI we could visualize progressive structural damages, such as edema and inflammatory lesions as well as bone erosion and formation in the lumbar spine over time. Post-mortem micro-CT confirmed this bone remodeling.

In this pilot study, only one sampling time was used to compare MRI, micro-CT and histology. MRI showed the inflammatory response occurring along ligaments, then expanding to soft tissues and bone, and the formation of bone-like structures over time, demonstrating the progressive nature of this pathology. Even if more extensive studies are needed to investigate the relationship between inflammation in the spine and bone remodeling, the development of progressive damage observed in vivo indicated a correlation with histopathological features of AS.

The present study including three complementary approaches clearly demonstrates the potential of histology in the demonstration of pathologic features provided by non-invasive imaging. Moreover, our data suggest that in
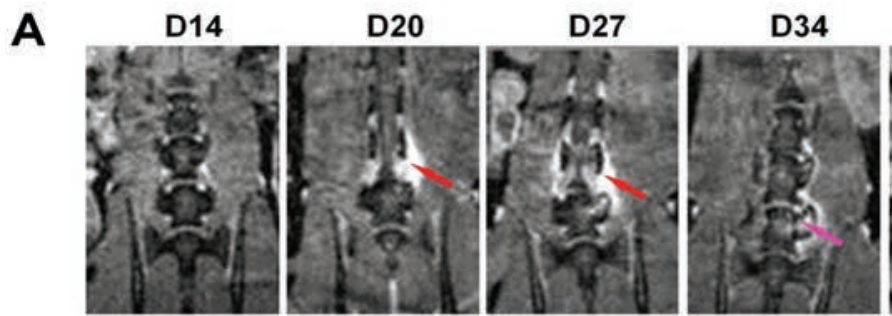

D41
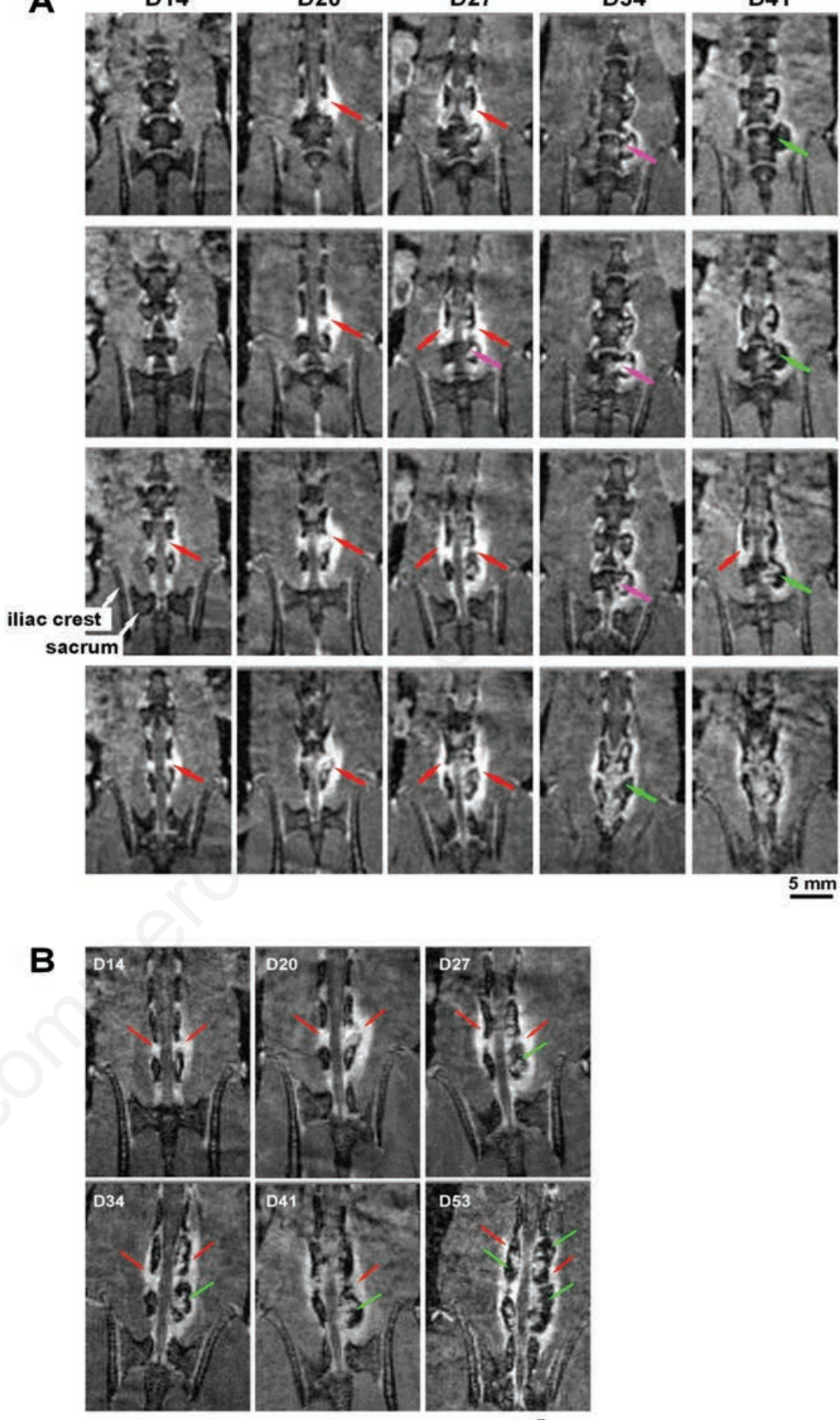

$5 \mathrm{~mm}$

Figure 2. CFA-induced effects at the levels L5-L6 of the lumbar spine depicted by MRI acquisitions performed at day 14 (D14), day 20 (D20), day 27 (D27), day 34 (D34) and day 41 (D41) after CFA administration. A) Four slices from 3D gradient-echo datasets at approximately the same anatomical location are depicted per time point. B) Gradientecho images at approximately the same anatomical location to highlight the formation of bone-like tissue from day 27 onwards; red arrows, inflammation; magenta arrows, inflammation within the bone, tentatively assigned to areas of bone erosion; green arrows, formation of bone-like tissue. Parameters for gradient-echo imaging at each time point: 3D fat-suppressed acquisition, echo time/repetition time: $2.5 / 57 \mathrm{~ms}$, field-of-view: $80 \times 40 \times 20 \mathrm{~mm}^{3}$, matrix: $256 \times 128 \times 96$, voxel size: $312 \times 312 \times 208 \mathrm{~mm}^{3}, 1$ average, acquisition time $11 \mathrm{~min} 40.4 \mathrm{~s}$. 
A
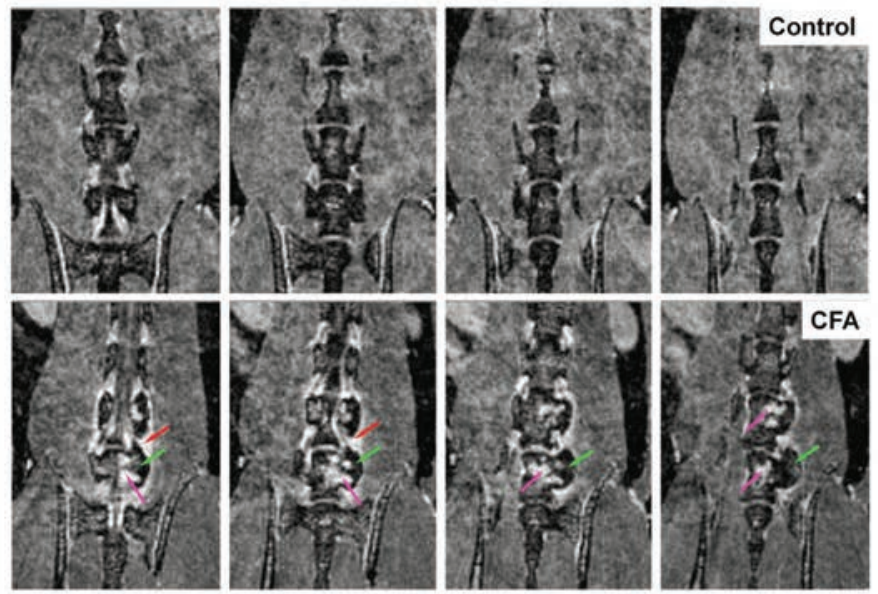

B
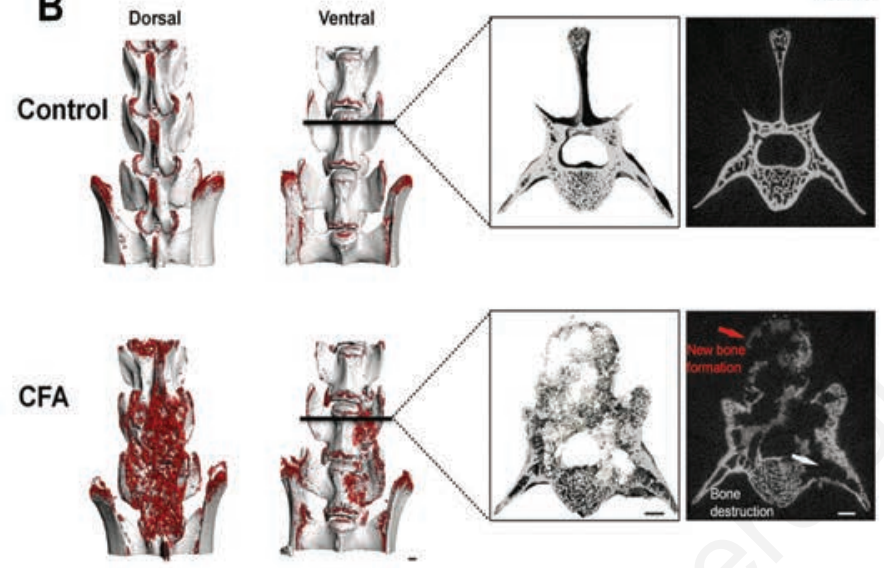

C
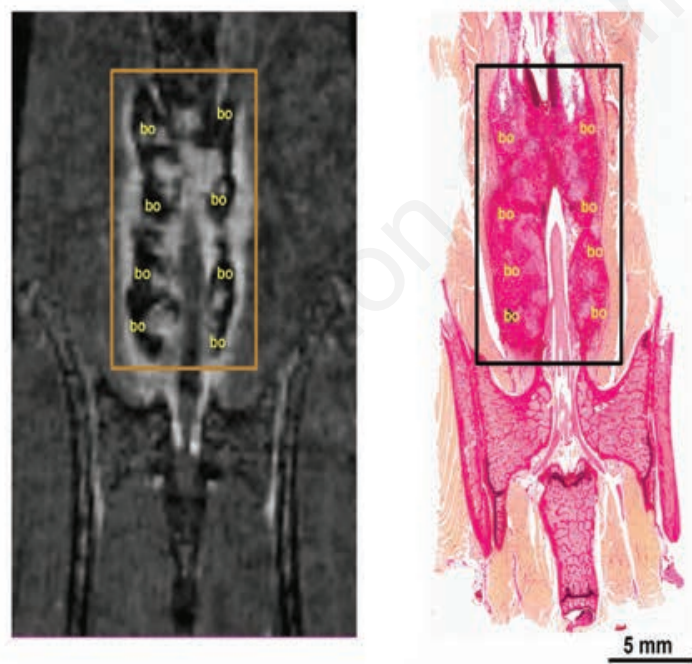

Figure 3. Images of the lumbar spine of the rat obtained in vivo by gradient-echo MRI and ex vivo using micro-CT. A) Four slices extracted from 3D MRI datasets acquired respectively from a control animal and from a rat at day 60 post-inoculation of CFA; red arrows, inflammation; magenta arrows, inflammation within the bone, tentatively assigned to areas of bone erosion; green arrows, formation of bone-like tissue. B) 3D segmented micro-CT images from excised spines of the same animals and higher magnification 3D cross-section through lumbar vertebra $\mathrm{L} 5$ (boxed) as well a corresponding $2 \mathrm{D}$ single slice (dark background); segmentation threshold: 150 (red), 280 (grey bone); scale bars: $1 \mathrm{~mm}$. C) Comparison of MRI and histology of the lumbar spine (L5-L6 level) of the CFA-rat. Bone-like (bo) structure formation as depicted by MRI (left panel) corresponds to the newly formed bone as revealed by histochemical AFPS staining (right). vivo imaging in this AS model may be used to validate the use of imaging in translational activities as therapies are moved into patient studies. Since AS has a very long disease development in humans, ${ }^{27}$ having access to well-characterized imaging readouts for early diagnosis becomes increasingly essential for therapy intervention studies..$^{28,29}$

\section{References}

1. Sieper J, Braun J, Rudwaleit M, Boonen A, Zink A. Ankylosing spondylitis: an overview. Ann Rheum Dis 2002;61(Suppl 3):iii8-18.

2. Australo-Anglo-American Spondyloarthritis Consortium (TASC), Reveille JD, Sims AM, Danoy P, Evans DM, Leo P, et al. Genome-wide association study of ankylosing spondylitis identifies non-MHC susceptibility loci. Nat Genet 2010;42:123-7.

3. Karaderi T, Harvey D, Farrar C, Appleton LH, Stone MA, Sturrock RD, et al. Association between the interleukin 23 receptor and ankylosing spondylitis is confirmed by a new UK case-control study and meta-analysis of published series. Rheumatology (Oxford) 2009;48:386-9.

4. Baraliakos X, Landewe R, Hermann KG, Listing J, Golder W, Brandt $\mathrm{J}$, et al. Inflammation in ankylosing spondylitis: a systematic description of the extent and frequency of acute spinal changes using magnetic resonance imaging. Ann Rheum Dis 2005;64:730-4.

5. Feldtkeller E, Khan MA, van der Heijde D, van der Linden S, Braun J. Age at disease onset and diagnosis delay in HLA-B27 negative vs. positive patients with ankylosing spondylitis. Rheumatol Int 2003;23:61-6.

6. Boonen A, Severens JL. Ankylosing spondylitis: what is the cost to society, and can it be reduced? Best Pract Res Clin Rheumatol 2002;16:691-705.

7. Anandarajah A, Ritchlin CT. Treatment update on spondyloarthropathy. Curr Opin Rheumatol 2005;17:247-56.

8. Rudwaleit M, Listing J, Brandt J, Braun J, Sieper J. Prediction of a major clinical response (BASDAI 50) to tumour necrosis factor alpha blockers in ankylosing spondylitis. Ann Rheum Dis 2004;63:665-70.

9. Baraliakos X, Listing J, Haibel H, Sieper J, Braun J. Vertebral erosions associated with spinal inflammation in patients with ankylosing spondylitis identified by magnetic resonance imaging: changes after 2 years of tumor necrosis factor inhibitor therapy. J Rheumatol 2013;40:1891-6.

10. Breban M. HLA-B27 transgenic rats model. Ann Med Interne (Paris) 1998;149:139-41. 
11. Adarichev VA, Glant TT. Experimental spondyloarthropathies: animal models of ankylosing spondylitis. Curr Rheumatol Rep 2006;8:267-74.

12. Lories RJ. Animal models of spondyloarthritis. Curr Opin Rheumatol 2006;18: 342-6.

13. Braem K, Lories RJ. Insights into the pathophysiology of ankylosing spondylitis: contributions from animal models. Joint Bone Spine 2012;79:243-8.

14. Zhang Y, Shi S, Ciurli C, Poole AR. Animal models of ankylosing spondylitis. Curr Rheumatol Rep 2002; 4:507-12.

15. Oostveen J, Prevo R, den Boer J, van de Laar M. Early detection of sacroiliitis on magnetic resonance imaging and subsequent development of sacroiliitis on plain radiography. A prospective, longitudinal study. J Rheumatol 1999; 26:1953-8.

16. Blum U, Buitrago-Tellez C, Mundinger A, Krause T, Laubenberger J, Vaith $\mathrm{P}$, et al. Magnetic resonance imaging (MRI) for detection of active sacroiliitis - a prospective study comparing conventional radiography, scintigraphy, and contrast enhanced MRI. J Rheumatol 1996;23: 2107-15.

17. Appel H, Loddenkemper C, Grozdanovic Z, Ebhardt H, Dreimann M, Hempfing A, et al. Correlation of histopathological findings and magnetic resonance imaging in the spine of patients with ankylosing spondyli- tis. Arthritis Res Ther 2006; 8:R143.

18. Ballet S, Conrath M, Fischer J, Kaneko T, Hamon M, Cesselin F. Expression and Gprotein coupling of mu-opioid receptors in the spinal cord and dorsal root ganglia of polyarthritic rats. Neuropeptides 2003;37: 211-9.

19. Iwamoto T, Takasugi Y, Higashino H, Ito H, Koga Y, Nakao S. Antinociceptive action of carbamazepine on thermal hypersensitive pain at spinal level in a rat model of adjuvant-induced chronic inflammation. J Anesth 2011;25:78-86.

20. Gouret C, Mocquet G, Raynaud G. Use of Freund's adjuvant arthritis test in antiflammatory drug screening in the rat: value of animal selection and preparation at the breeding center. Lab Anim Sci 1976;26:281-7.

21. Dawson J, Gustard S, Beckmann N. Highresolution three-dimensional magnetic resonance imaging for the investigation of knee joint damage during the time course of antigen-induced arthritis in rabbits. Arthritis Rheum 1999;42:119-28.

22. Verdenius HH, Alma L. A quantitative study of decalcification methods in histology. J Clin Pathol 1958; 11:229-36.

23. Kalscheur VL. Bone and cartilage in osteoarthritis: a proteoglycan stain. HistoLogic Techn Bull Histotechnol 2001; XXXIV:14-5.
24. Gomori G. Aldehyde-fuchsin: a new stain for elastic tissue. Am J Clin Pathol 1950; 20:665-6.

25. Dijkstra CD, Dopp EA, van den Berg TK, Damoiseaux JG. Monoclonal antibodies against rat macrophages. J Immunol Methods 1994;174:21-3.

26. Damoiseaux JG, Dopp EA, Calame W, Chao D, MacPherson GG, Dijkstra CD. Rat macrophage lysosomal membrane antigen recognized by monoclonal antibody ED1. Immunology 1994;83:140-7.

27. Tan S, Yao J, Flynn JA, Yao L, Ward MM. Quantitative syndesmophyte measurement in ankylosing spondylitis using CT: longitudinal validity and sensitivity to change over 2 years. Ann Rheum Dis 2015;74:437-43.

28. Baeten D, Baraliakos X, Braun J, Sieper J, Emery P, van der Heijde D, et al. Anti-interleukin-17A monoclonal antibody secukinumab in treatment of ankylosing spondylitis: a randomised, double-blind, placebo-controlled trial. Lancet 2013;382: 1705-13.

29. Baraliakos X, Borah B, Braun J, Baeten D, Laurent D, Sieper J, et al. Long-term effects of secukinumab on MRI findings in relation to clinical efficacy in subjects with active ankylosing spondylitis: an observational study. Ann Rheum Dis 2016;75:408-12. 